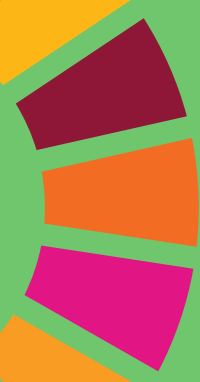

\title{
LA IMPORTANCIA DE
} LA INCLUSIÓN

\section{FINANCIERA EN MÉXICO}

\section{PARA ALCANZAR LOS ODS}

\section{THE IMPORTANCE OF FINANCIAL INCLUSION IN MEXICO TO THE ACHIEVEMENT OF THE SDGS}

\author{
María del Carmen Dircio Palacios Macedo \\ Universitat Jaume I \\ maricarmen.dircio@gmail.com
}

Fecha recepción artículo: 12/03/2021 • Fecha aprobación artículo: 09/06/2021

\section{RESUMEN}

El objetivo del presente artículo es abordar la importancia de la promoción de la inclusión financiera en México para la consecución de los ODS y documentar diversos avances en torno a ello. En primer lugar, se exponen las relaciones que se han encontrado con respecto al vínculo de la inclusión financiera y diferentes aspectos del desarrollo económico. En segundo lugar, se da un panorama del rezago en la inclusión financiera en México, se presentan algunos avances recientes, y cómo estos promueven la consecución de los ODS. La inclusión financiera se encuentra explícitamente incluida dentro del ODS 8, en la meta 8.10. Pero además, es un medio para conseguir una buena parte de los ODS. Reconociendo la problemática de la falta de inclusión financiera, en México se han seguido varias estrategias, políticas públicas y cambios en regulaciones, tendientes a promover la inclusión financiera. Se da cuenta de esto, además de los esfuerzos por cuantificar el rol de los bancos en torno al cumplimiento de los ODS a través de una alianza entre el Programa de las Naciones Unidas (PNUD) en México y la Asociación de Bancos de México (ABM).

${ }^{1}$ Economista mexicana especialista en temas de competencia económica, análisis de mercados y desarrollo económico. Licenciada en Economía por la Universidad de las Américas Puebla- México. Maestra en Economía Aplicada por la Universidad Popular Autónoma de Puebla, México. Máster en Economía por la Universitat Jaume I. Se desempeñó como Directora de Área de la Dirección General de Estudios Económicos de la Comisión Federal de Competencia Económica de México. Ha sido consultor independiente en temas de competencia económica. Actualmente es estudiante del Doctorado en Economía y Empresa, en UJI 
Palabras clave: Finanzas sostenibles Mexico, Inclusión financiera y desarrollo económico; Inclusión financiera ODS, Agenda 2030

\section{SUMMARY}

The objective of this article is to address the importance of promoting financial inclusion in Mexico for the achievement of the SDGs, and to document various advances in this regard. In the first place, the relationships that have been found regarding the link between financial inclusion and different aspects of economic development are presented. Second, there is an overview of the lag in financial inclusion in Mexico, some recent advances are documented, and how these promote the achievement of the SDGs. Financial inclusion is explicitly included within SDG 8, in target 8.10. But it is also a means to achieve many of the other SDGs. Recognizing the problem of the lack of financial inclusion, in Mexico several strategies, public policies and changes in regulations have been followed, aimed at promoting financial inclusion. I present these advances, in addition to the efforts to quantify the role of banks in meeting the SDGs through an alliance between the United Nations Program (UNDP) in Mexico and the Association of Banks of Mexico (ABM).

Keywords: Sustainable finance Mexico, Financial inclusion and development, Financial inclusion and SDG, 2030 Agenda

\section{INTRODUCCIÓN}

Los Objetivos de Desarrollo Sostenible (ODS) fueron establecidos en 2015 como parte de la Agenda 2030 para el Desarrollo Sostenible de la ONU, comprendiendo 17 ODS y 169 metas de carácter transversal, confiriendo un papel mucho más significativo al sector privado. Se considera que sin la participación de este, difícilmente se lograrán los objetivos planteados para el 2030, de hecho, 88 de las 169 metas establecidas en la Agenda 2030 requiere un rol activo del sector privado para su cumplimiento. (Villasuso \& PNUD, 2020)

México se ha comprometido con el cumplimiento de los ODS y ha avanzado en los años recientes, con la participación del gobierno y del sector privado. Sin embargo, aún existen rezagos y retos importantes en torno al cumplimiento de la Agenda 2030. Por ejemplo, en México sigue habiendo un $41.9 \%$ de la población en situación de pobreza y un $7.4 \%$ de la población en pobreza extrema. Asimismo, el 16.9\% de la población tiene rezago educativo y el $57.3 \%$ tiene carencia por acceso a la seguridad social. (CONEVAL, 2019) 
Adicionalmente en México, hay un rezago importante en la inclusión financiera de una parte de la población, que es especialmente severo en algunas regiones y en poblaciones rurales, en transición y semiurbanas, y en cierto tipo de servicios. De ahí la relevancia de abordar la necesidad de avance en la inclusión financiera para la consecución de los ODS.

El avance hacia la inclusión financiera de la población, contribuye a los ODS por la relación que existe entre inclusión financiera y desarrollo económico. La inclusión financiera de hecho está contemplada como una meta en el ODS 8. Pero además, como se argumentará en el presente artículo, contribuye y está vinculada a la consecución de muchos de los demás ODS.

El objetivo general del presente artículo es abordar la importancia de la promoción de la inclusión financiera en México para la consecución de los ODS, y documentar diversos avances en torno a ello. Para cumplir con el objetivo, en la primera sección se exponen las relaciones que se han encontrado en artículos de investigación, tanto la argumentación teórica como algunas evidencias empíricas, respecto al vínculo entre desarrollo financiero, inclusión financiera y diversas variables de crecimiento y desarrollo económico. En la segunda sección se da un panorama del rezago en la inclusión financiera en México, se exponen algunos avances recientes, y cómo estos promueven la consecución de los ODS. Por último, se presentarán las conclusiones y retos para la inclusión financiera y su impacto en los ODS.

\section{INCLUSIÓN FINANCIERA, DESARROLLO ECONÓMICO Y LOS ODS}

\subsection{DESARROLLO FINANCIERO, INCLUSIÓN FINANCIERA Y DESARROLLO ECONÓMICO}

Para establecer cómo la inclusión financiera promueve los ODS, previamente haré referencia muy brevemente a algunos artículos que respaldan la importancia del sector financiero en el crecimiento y desarrollo económico.

En primer lugar se presentan algunas definiciones. Para cuantificar la importancia del sector financiero en la economía, se ha usado el concepto de desarrollo financiero, el cual se mide mediante indicadores de profundización financiera, a nivel macro, como lo son la capitalización de mercado del mercado de valores o la relación entre el crédito y el producto interno bruto (PIB) de un país.

La inclusión financiera puede definirse como el acceso de las empresas y los hogares a servicios financieros formales adecuados y a precios razonables que satisfagan sus necesidades. (Beck, 2016)

Por su parte Demirguc-Kunt, Klapper, and Singer (2017) consideran que la inclusión financiera significa que la población adulta tenga acceso y pueda hacer uso efectivamente de un rango apropiado de servicios financieros. Y que tales servicios sean ofrecidos responsablemente y de forma segura al consumidor, y de forma sostenible por el proveedor en un ambiente bien regulado.

Existe una extensa literatura que ha establecido una relación positiva entre desarrollo financiero, profundidad financiera y el crecimiento económico. Sin embargo, de acuerdo a (Demirguc-Kunt et al., 2017), aún es necesaria mayor investigación sobre la relación entre inclusión financiera, desigualdad y crecimiento macroeconómico. 
La dificultad de establecer relaciones claras radica en que hay varios canales y formas mediante las cuales la inclusión financiera incide en diferentes aspectos del desarrollo económico. Debe considerarse además, que tanto la inclusión financiera como el desarrollo económico son conceptos multidimensionales.

Varios investigadores han abordado teóricamente el asunto y han demostrado el vínculo entre desarrollo financiero y desarrollo económico. Beck, Demirguc-Kunt, and Martinez Peria (2007) exponen la teoría que sustenta la importancia de un acceso amplio a los servicios financieros para el desarrollo económico. En primer lugar, de acuerdo a lo examinado por Levine (2005), los autores argumentan sobre la importancia de un sistema financiero bien desarrollado para el combate de la pobreza. Cuando se tiene un mercado financiero con imperfecciones estas afectan mucho más a los empresarios pobres o pequeños para financiar proyectos de inversión de alto rendimiento, lo que tiene consecuencias adversas para el crecimiento y el alivio de la pobreza. En segundo lugar, los autores argumentan que uno de los canales a través de los cuales el desarrollo financiero fomenta el crecimiento económico es a través de la entrada de nuevas empresas, y la falta de desarrollo financiero impide esta entrada. En tercer lugar, se señala que el acceso a la financiación también puede tener efectos importantes sobre el progreso tecnológico y la generación de ideas.

Por otra parte, hay diversos estudios que demuestran los vínculos de diversos aspectos de inclusión, en diferentes variables de desarrollo. Sin pretender ser exhaustiva, se citan algunos ejemplos.

Un estudio que demuestra el vínculo entre inclusión y variables de desarrollo económico para México es el de Bruhn and Love (2014). Los investigadores analizan la apertura de Banco Azteca en México, que fue como un "experimento natural" en el que más de 800 oficinas bancarias abrieron casi simultáneamente en tiendas de bienes de consumo durables preexistentes. El principal resultado que encontraron fue un efecto considerable del acceso al financiamiento sobre la actividad del mercado laboral y los niveles de ingresos, especialmente entre las personas de bajos ingresos y las situadas en zonas con menor penetración bancaria preexistente.

Por otra parte, en un estudio transversal de países, realizado por Aslan, Deléchat, Newiak, and Yang (2017), basado en la encuesta Findex (Demirgüç-Kunt Asli, Klapper Leora, Singer Dorothe, Saniya Ansar, \& Jake, 2018), se demuestra que la desigualdad en el acceso a los servicios financieros está relacionada positiva y significativamente con la desigualdad del ingreso.

Dabla-Norris, Ji, Townsend, and Unsal (2015) por su parte, desarrollan un modelo de equilibrio general con fundamentos micro, con agentes heterogéneos, para identificar las limitaciones a la inclusión financiera. Evalúan cuantitativamente los impactos políticos de relajar cada una de estas restricciones por separado y en combinación sobre el PIB y la desigualdad. Su estudio considera seis países con distintos grados de desarrollo económico.

Tomando en cuenta que la inclusión financiera es un concepto multidimensional, algunos artículos evalúan sus efectos en el desarrollo tomando por ejemplo un índice compuesto de inclusión. Por ejemplo Koomson, Villano, and Hadley (2020) examinan el efecto de la inclusión financiera sobre la pobreza y la vulnerabilidad a la pobreza utilizando un índice de inclusión financiera multidimensional para Ghana. 


\subsection{LA RELACIÓN ENTRE INCLUSIÓN FINANCIERA Y EL CUMPLIMIENTO DE LOS ODS}

La inclusión financiera está contemplada dentro del ODS 8, que es:

Promover el crecimiento económico sostenido, inclusivo y sostenible, el empleo pleno y productivo y el trabajo decente para todos. (ONU, 2017)

Una de las metas de este objetivo, la 8.10 contempla:

8.10 Fortalecer la capacidad de las instituciones financieras nacionales para fomentar y ampliar el acceso a los servicios bancarios, financieros y de seguros para todos

Los indicadores para el cumplimiento de estos objetivos son los siguientes:

8.10.1 a) Número de sucursales de bancos comerciales por cada 100.000 adultos y b) número de cajeros automáticos por cada 100.000 adultos

8.10.2 Proporción de adultos (apartir de 15 años de edad) que tienen una cuenta en un banco u otra institución financiera o un proveedor de servicios de dinero móvil

(ONU, 2017)

Sin embargo, más allá de estar contemplada en el ODS 8, como ya se argumentó en el anterior apartado, hay varios canales por los cuales la inclusión financiera puede incidir en variables del desarrollo económico, y de hecho es clave para alcanzar muchos de ellos.

Un artículo que trata específicamente de cómo la inclusión financiera puede ayudar a la consecución de los ODS, cuáles de ellos, es el de Klapper, El-Zoghbi, and Hess (2016). Estos autores se basan en estudiar la investigación empírica para varios países al respecto, recopilan estos estudios y a partir de allí argumentan que algunos objetivos son promovidos de manera directa por la inclusión financiera, mientras que, para otros objetivos, existen motivos teóricos para considerar que la inclusión financiera puede ayudar a promoverlos de manera indirecta, aunque faltan aún más estudios al respecto. Argumentan que la inclusión financiera puede incidir de hecho en la mayor parte de los ODS, aunque la evidencia empírica no es suficiente en el caso de algunos.

Se mencionarán algunos de los argumentos sobre la relación de la inclusión financiera y la consecucion de los ODS en particular, siguiendo a Klapper et al. (2016).

Con relación al ODS 1, que es poner fin a la pobreza en todas sus formas y en todo el mundo, el argumento es que cuando las personas están incluidas en el sistema financiero, están en mejores condiciones de salir de la pobreza invirtiendo en negocios o educación. De hecho se menciona explícitamente la importancia del acceso a los servicios financieros, en la meta 1.4:

1.4 De aquí a 2030, garantizar que todos los hombres y mujeres, en particular los pobres y los vulnerables, tengan los mismos derechos a los recursos económicos y acceso a los servicios básicos, la propiedad y el control de la tierra y otros bienes, la herencia, los recursos naturales, las nuevas tecnologías apropiadas y los servicios financieros, incluida la microfinanciación (ONU, 2017) 
Respecto al ODS 2, que es poner fin al hambre, lograr la seguridad alimentaria y la mejora de la nutrición y promover la agricultura sostenible, Klapper et al. (2016) argumentan que la falta de acceso a crédito y seguros impide que los agricultores realicen inversiones que podrían aumentar el rendimiento de los cultivos y fortalecer la seguridad alimentaria. Sin embargo, la base de datos Global Findex muestra que solo el 10\% de los residentes rurales en los países en desarrollo utiliza crédito formal y solo la mitad tiene una cuenta. (Demirgüç-Kunt Asli et al., 2018)

Por lo que tiene que ver con el ODS 3, que es Garantizar una vida sana y promover el bienestar de todos a todas las edades, se argumenta que la inclusión financiera mejora la salud al brindar a las personas la capacidad de administrar los gastos médicos y recuperarse de una crisis de salud. Por ejemplo, los servicios financieros como el seguro médico pueden proporcionar un canal formal para mitigar las emergencias de salud.

Con relación al ODS 4, que es el Garantizar una educación inclusiva y equitativa de calidad y promover oportunidades de aprendizaje permanente para todos, el argumento es que lograr una educación de calidad depende de que las personas tengan la capacidad de invertir en oportunidades de aprendizaje. Por ejemplo, los productos de ahorro ayudan a las familias a planificar y administrar los gastos de educación.

Respecto al ODS 5, Lograr la igualdad de género y empoderar a todas las mujeres y las niñas, se considera que los servicios financieros ayudan a las mujeres a afirmar su poder económico, que es clave para promover la igualdad de género. Inclusive se argumenta que la inclusión financiera de las mujeres respalda muchos objetivos de desarrollo más allá del ODS 5 , puesto que se ha demostrado que cuando las finanzas son controladas por mujeres, es más probable que se gasten en necesidades básicas, como alimentos y agua, así como en bienestar infantil, incluidas las colegiaturas escolares y cuidado de la salud. (Klapper et al. (2016)

Por lo que tiene que ver con el ODS 6 y ODS 7, Garantizar la disponibilidad y la gestión sostenible del agua y el saneamiento para todos (ODS 6) y Energía (ODS 7), se argumenta que hay muchas razones para considerar que es probable que las innovaciones en los servicios financieros digitales aceleren el acceso a estos recursos, aunque la literatura aún no documenta definitivamente este impacto.

Con relación al ODS 8, Promover el crecimiento económico sostenido, inclusivo y sostenible, el empleo pleno y productivo y el trabajo decente para todos, Klapper et al. (2016) argumentan que el acceso a instituciones y productos financieros permite a las personas obtener mayores rendimientos sobre el capital. Esto conduce a aumentos en sus ingresos y, en consecuencia, afecta el crecimiento económico. Por ejemplo, un estudio de Bruhn and Love (2014), demuestra que para México los ingresos aumentaron en un 7\% en áreas donde se abrieron rápidamente oficinas bancarias en más de 800 tiendas minoristas.

Respecto al ODS 9, Construir infraestructuras resilientes, promover la industrialización inclusiva y sostenible y fomentar la innovación, se considera que para cumplir este objetivo se requiere un fácil acceso al crédito y otros servicios financieros que facilitan la inversión.

Por lo que tiene que ver con el ODS 10 y ODS 16, que se relacionan con construir sociedades equitativas y pacíficas, Klapper et al. (2016) señalan que el acceso a los servicios financieros puede permitir a las persona estar mejor posicionadas para tener éxito económicamente y construir una vida decente, lo que en última instancia facilita la reducción de la desigualdad (ODS 10) y la promoción de la paz (ODS 16). 


\section{EL REZAGO EN LA INCLUSIÓN FINANCIERA EN MÉXICO, LAS ESTRATEGIAS Y POLÍTICAS IMPLEMENTADAS, Y LOS AVANCES HACIA LOS ODS}

Si bien ha habido un crecimiento en los servicios bancarios en los últimos años en México, existe un rezago en la inclusión financiera de una parte de la población. En muchas localidades no existen los canales de infraestructura requeridos, pero además, en muchos casos aunque estos canales estén disponibles, no hay suficiente uso de ellos.

En México existe una parte de su población que está financieramente excluida, especialmente en las zonas rurales. Una buena parte de los municipios más pobres y menos poblados no tienen oficinas bancarias, aunque en algunos casos, las personas pueden tener acceso a alguna oficina bancaria en municipios cercanos. En otros casos tienen acceso a otros canales, como pueden ser los corresponsales o cajeros. Existe una gran diversidad en los niveles de inclusión en los municipios de México, existiendo localidades de muy alta inclusión y otras de muy baja inclusión.

La Gráfica 1 muestra diferentes indicadores de inclusión financiera para México, junto con el promedio de los países de la OCDE, para el año 2017 (último año disponible). Los datos son del Global Findex del Banco Mundial del 2017. (Demirgüç-Kunt Asli et al., 2018) Esta encuesta muestra que sólo el 35\% de la población en México tiene una cuenta (ya sea en forma exclusiva o conjunta con otra persona) en un banco u otro tipo de institución financiera, mientras que para los países de la OCDE este porcentaje se acerca al 95\% (en promedio). Asimismo, solo el 32\% de la población en México informa haber pedido prestado dinero (individualmente o junto con otra persona) por cualquier motivo y de cualquier fuente en los últimos 12 meses. Finalmente, sólo el $9.5 \%$ de la población mexicana reporta tener tarjeta de crédito, un porcentaje mucho más bajo que el promedio de los países de la OCDE (57\%).

Gráfica 1. Indicadores de inclusión financiera para México y OCDE (2017)

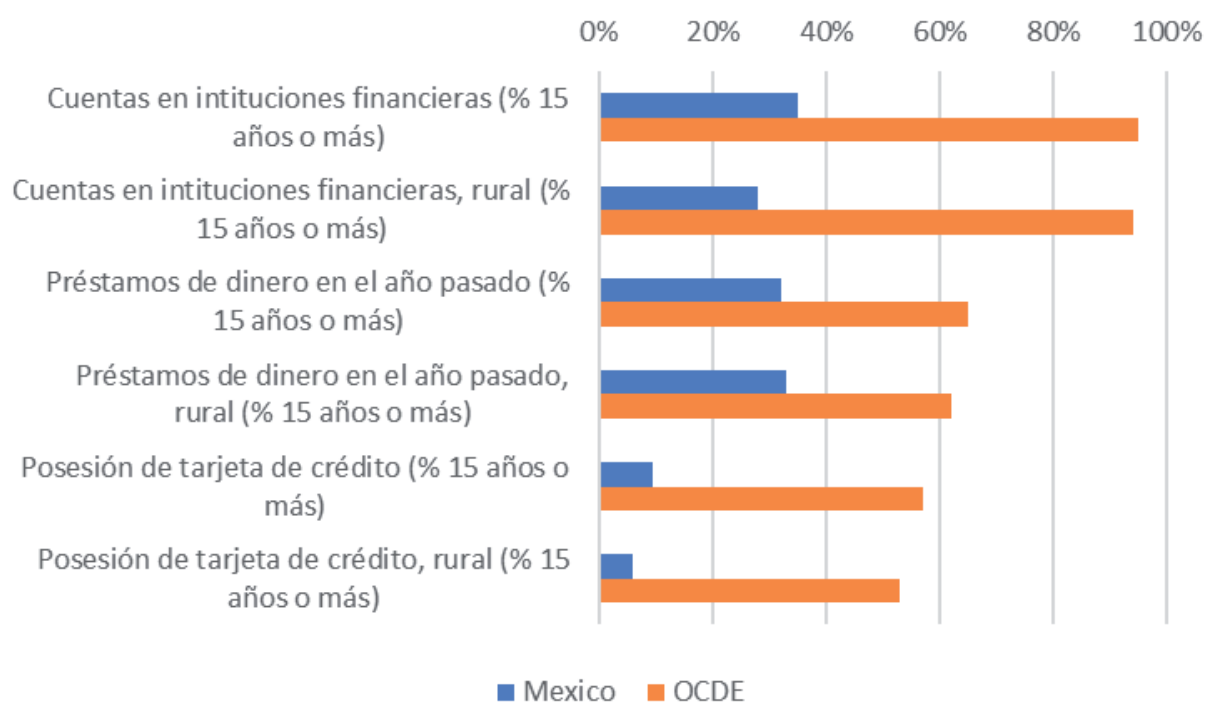

Fuente: Datos de Global Findex del Banco Mundial del 2017. (Demirgüç-Kunt Asli et al., 2018) 
Una propuesta de solución para esta falta de acceso físico puede venir de un mayor acceso electrónico o móvil, sin embargo, esta opción presenta dificultades para poder implementarse en regiones apartadas de México. Dos obstáculos importantes son la falta de conexión de internet en una buena parte de los municipios, que son precisamente los que experimentan exclusión financiera, así como también un rezago en educación financiera que es necesaria para que las personas utilicen y confíen en estos nuevos medios.

Con el propósito de explicar esta falta de canales de acceso e indagar en cuáles son los factores que inciden en que los municipios en México tengan o no infraestructura, Cruz-García, Dircio Palacios Macedo, and Tortosa-Ausina (2021) propusimos modelos de regresión logística. Se midió la inclusión financiera utilizando dos variables alternativas: la existencia en el municipio con al menos una oficina bancaria o la existencia con al menos un cajero automático. Las variables explicativas que se utilizaron fueron factores de oferta y demanda que impliquen una mayor probabilidad de inclusión, como lo son la población, la densidad de población y el Índice de Desarrollo Humano (IDH). También se corrieron modelos para las diferentes regiones de México y tipos de población.

Se encontró que el tamaño de la población y el IDH tienen una relación directa y significativa con la probabilidad de un municipio de estar incluido, mientras que respecto a la densidad de población la relación no es tan clara y en algunas regiones y tipos de población no es significativa.

Por tanto tenemos que un bajo nivel de desarrollo humano de un municipio, o un tamaño pequeño de pobladores hace menos atractivo para un banco el establecer una oficina u otro canal de acceso. Hay bajos incentivos para los bancos de establecerse en estos municipios, pero a la vez son estos mismos los que requieren ser incluidos, y en donde prevalecen niveles de pobreza, falta de acceso a la salud, y demás rezagos en el desarrollo humano, en los cuales es necesario un avance hacia el cumplimiento de los ODS.

Por otra parte, además de este rezago en los puntos de acceso, hay también un rezago en el uso de los servicios financieros. La causas de ello pueden ser una falta de cultura financiera, y diversas razones de autoexclusión. De hecho, ha habido una tendencia a la persistencia del efectivo para muchas transacciones, aún cuando ha habido un crecimiento importante en el uso de instrumentos de pago digitales, como lo son el uso de tarjetas de crédito y débito, transferencias electrónicas de fondos y banca móvil. Del Angel (2016) sostiene que la baja inclusión financiera y la actividad económica informal pueden considerarse las principales causas de esta tendencia.

\subsection{ESTRATEGIAS Y COMPROMISOS EN TORNO A LA INCLUSIÓN FINANCIERA}

Reconociendo la problemática de falta de inclusión financiera de una parte importante de la población, se han buscando soluciones en México dando varios pasos. En 2008, la Comisión Nacional Bancaria y de Valores (CNBV), regulador mexicano del sector, contempló a la inclusión financiera como un objetivo estatutario formal en su plan estratégico. En 2009, México se convirtió en "Miembro Principal" de la Alianza para la Inclusión Financiera (AFI, por sus siglas en inglés) y en 2011, miembro de la Declaración Maya firmando varios compromisos para incrementar la inclusión financiera. 
La AFI fue fundada en 2008 como la primera red de intercambio de conocimiento global diseñada exclusivamente para las autoridades de inclusión financiera de los países en desarrollo. Dentro de esta red fue creado "El Grupo de Trabajo para la Medición de la Inclusión Financiera", con el fin de compartir la experiencia en la recolección y medición de datos de inclusión financiera.²

La Declaración Maya, elaborada y apoyada por los miembros de la AFI, es el primer conjunto global de compromisos medibles para aumentar el acceso a los servicios financieros formales para los 2,5 mil millones de personas sin bancarizar en el mundo. ${ }^{3}$

En el contexto de la Declaración Maya, México se comprometió a establecer una estrategia nacional de inclusión financiera, facilitar la provisión de servicios financieros móviles, tener un sistema robusto de datos y medición implementado mediante el lanzamiento de una encuesta del lado de la demanda y la publicación de información trimestral sobre transacciones electrónicas, y tener una sucursal bancaria o un agente bancario en cada municipio al 2014. Se han cumplido los tres primeros compromisos:

- En 2013 se publicaron los resultados de la primera Encuesta Nacional de Inclusión Financiera (ENIF) y, en 2014, se aprobó la Reforma Financiera con el objetivo de fortalecer el entorno regulatorio, aumentar la competencia y reducir el costo de endeudamiento especialmente para pequeñas y medianas empresas (PYMES).

- En 2016, se finalizó la Estrategia Nacional de Inclusión Financiera.

- La medición de datos de inclusión financiera, mediante el diseño de bases de datos e indicadores de inclusión financiera, es otro elemento prominente de la agenda. En México la CONAIF publica cuatrimestralmente bases de datos de inclusión financiera, con información que aportan los bancos al regulador.

Sin embargo, respecto a los puntos de acceso, hay pendientes importantes. En 2015, el 31\% de los 2457 municipios no tenían puntos de acceso financiero. (OECD, 2017) Aunque se ha avanzado desde 2015 hasta la presente fecha, aún hay muchos municipios sin ningún punto de acceso. Los avances se muestran en la siguiente tabla.

Tabla 1. Porcentaje de municipios con al menos un punto de acceso

\begin{tabular}{|c|c|c|c|c|c|c|}
\hline 2014 & 2015 & 2016 & 2017 & 2018 & 2019 & Meta 2024 \\
\hline 69.7 & 68.9 & 72.5 & 73.4 & 76.6 & 78 & 89.6 \\
\hline
\end{tabular}

\footnotetext{
${ }^{2}$ https://www.cnbv.gob.mx/Inclusión/Paginas/Indicadores-AFI.aspx

${ }^{3} \mathrm{https}: / /$ www.findevgateway.org/es/publicacion/2013/11/ubicando-la-inclusion-financiera-en-el-mapa-mundial
} 


\subsection{POLÍTICAS RECIENTES QUE HAN PROMOVIDO LA INCLUSIÓN FINANCIERA EN MÉXICO}

Con el objeto de avanzar en la inclusión financiera, algunas políticas que se han implementado recientemente en México son las siguientes:

\section{a) La introducción y aumento de los corresponsales bancarios}

Los corresponsales son establecimientos comerciales, como tiendas minoristas o de conveniencia, farmacias y supermercados, que en virtud de un contrato prestan ciertos servicios financieros en nombre de los bancos. Estos servicios incluyen retirar efectivo, operaciones de depósito, el pago de préstamos y servicios públicos y el pago de cheques emitidos por el banco.

Los corresponsales bancarios comenzaron a operar en 2009. Desde entonces, el número de unidades de corresponsales bancarios ha superado el número de sucursales bancarias, y de esta manera ha sido clave para ampliar los puntos de acceso, aumentando así la cobertura geográfica. De acuerdo con la Base de Datos de Inclusión Financiera 2020, en ese año habían 1,74 oficinas por cada 10.000 adultos a nivel nacional, que habían decrecido -6\% en el periodo 2015-2020. Mientras tanto, los corresponsales eran 5,22 por cada 10,000 adultos a nivel nacional, y habían crecido 30\% en el periodo. (CONAIF, 2015, 2016, 2017, 2018, 2019, 2020)

Destaca entonces el crecimiento de corresponsales, que han dado cobertura a municipios en los que antes no había acceso. No obstante los corresponsales no ofrecen todos los servicios que están disponibles en las oficinas bancarias, por tanto pueden ser más bien complementarios a éstas últimas, y no cubrir por completo las necesidades de inclusión financiera requeridas por la población que atienden. Además de que en muchos casos no cubren municipios rurales o en transición, que son los más afectados por exclusión.

El aumento en los corresponsales como punto de acceso es una política que favorece la consecución del ODS 8, meta 8.10, aunque no esté contemplado explícitamente como un indicador.

\section{b) El impulso al sector de ahorro y crédito popular}

El sector de ahorro y crédito popular incluye cooperativas y microfinancieras, entre ellas las llamadas Socaps, Sofoles, Sofomes y Sofipos, que son entidades que tradicionalmente se han especializado en el segmento de menores ingresos. Ha habido cambios en su regulación en que se les autorizó a algunas que presten mayores servicios, y en otros casos se les reguló para que tuvieran más transparencia y sean más confiables para los usuarios, y así puedan incrementar los servicios bancarios ofrecidos. Estos sectores tienen una participación baja en el monto de financiamiento, representando alrededor de $0.8 \%$ del PIB en términos de activos. (Urbina Romero, 2021) No obstante, su rol puede ser importante en términos de inclusión financiera pues algunas Socaps y Sofipos operan en localidades donde no existe presencia de la banca tradicional y atienden a sectores poblaciones que por lo general, no son atendidos por otros intermediarios. Además han aumentado sus puntos de acceso, aunque no significativamente.

Por su rol en la inclusión financiera, se considera que la política de impulsar a estos intermediarios no financieros promueve la consecución del ODS 8, meta 8.10, aun cuando no esté contemplado explícitamente como un indicador a observar. 
c) CoDi

CoDi es una plataforma introducida en 2019 desarrollada por el Banco de México para facilitar las operaciones de pago y cobro a través de transferencias electrónicas, por medio de teléfonos móviles, que no tiene costo para el usuario. CoDi utiliza la tecnología de códigos QR y NFC. Esta plataforma ha crecido mucho desde su introducción. Mientras que en Diciembre de 2019 tenía 1.389 usuarios registrados, para Septiembre 2020 eran 18.121 usuarios. En ese mismo periodo de tiempo, las transferencias CoDi realizadas pasaron de ser 1.886l, a 27.934. ${ }^{4}$

Esta plataforma, al incentivar las transferencias electrónicas, promueve la consecución del ODS 8, meta 8.10.

\section{d) Banco del Bienestar}

Recientemente, el Gobierno mexicano ha impulsado la creación de bancos estatales en municipios sin cobertura previa, programa denominado Banco del Bienestar. Este programa ha tenido el objetivo de impulsar el crecimiento de oficinas bancarias, por medio de las cuales se realicen las transferencias de los programas gubernamentales, pero que también presten otros servicios como créditos. En el momento de su anuncio el proyecto contempló que, entre 2020 y 2021, fueran edificadas 2.700 sucursales de este banco.

Sin embargo este programa ha recibido varias críticas por no cumplir las expectativas y por su diseño dependiente de los fondos públicos. A poco más de un año del anuncio, el Banco del Bienestar cuenta apenas con 433 sucursales en operación, reporta un alto índice de morosidad del 19,17\% y una pérdida neta de 63.4 millones de pesos al primer trimestre de $2021 .{ }^{5}$

Estas nuevas oficinas, al no ser de la banca comercial, no incidirían en el indicador 8.10.1, del ODS 8, citado en el anterior apartado. Sin embargo, al fomentar la inclusión financiera, promueve la consecución del ODS 8, meta 8.10.

\subsection{AVANCES HACIA LA INCLUSIÓN FINANCIERA Y EL CUMPLIMIENTO DE LOS ODS EN MÉXICO}

Las siguientes gráficas muestran la evolución a nivel nacional de los indicadores de acceso en México desde el año 2015 en el que se adoptaron los compromisos de los ODS, a 2020. Todos los indicadores de las gráficas son por cada 10.000 adultos. Estas cifras al ser nacionales, no muestran los cambios en regiones o tipos de población excluidas, que sería importante para evaluar las avances en la inclusión. Sin embargo, dan un panorama general de cómo ha evolucionado el acceso financiero en años recientes. En la Gráfica 2 se observa que las oficinas bancarias comerciales han disminuido en el periodo, siendo esto acorde con una tendencia mundial, aunque en un país como México en que hay muchas localidades desatendidas, más bien se requiere una expansión. Las oficinas de la banca de desarrollo, cooperativas y microfinancieras han permanecido sin muchas modificaciones significativas en el periodo, a nivel nacional. Siendo el indicador de estas últimas mucho menor que el de la banca comercial. La cifra del número de oficinas de la banca comercial sí está contemplado como un indicador de la meta 8.10, que es el 8.10.1a), y lo que se observa es que no ha habido un avance en el periodo.

\footnotetext{
${ }^{4}$ https://www.banxico.org.mx/sistemas-de-pago/codi-cobro-digital-banco-me.html

${ }^{5}$ https://elpais.com/mexico/economia/2021-05-10/el-banco-del-bienestar-capta-mas-recursos-publicos-pese-a-su-alto-indicede-morosidad.html
} 
Gráfica 2. Oficinas bancarias por cada 10,000 adultos en México (2015-2020)

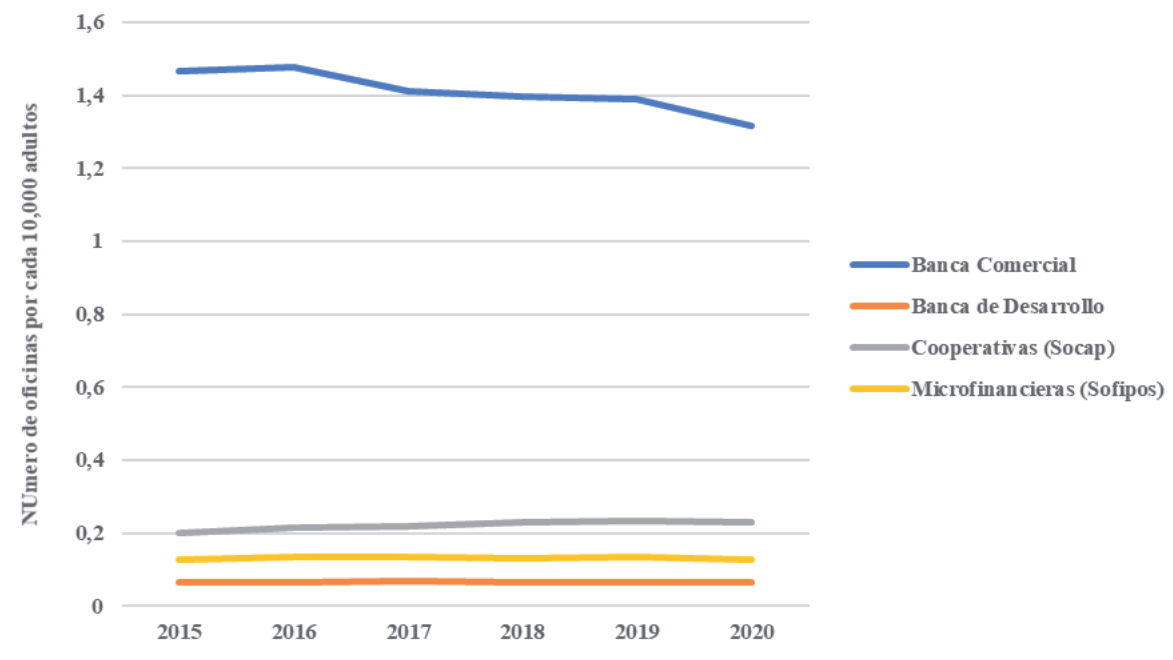

Fuente: Base de Datos de Inclusión Financiera, varios años (CONAIF, 2015, 2016, 2017, 2018, 2019, 2020).

Recuperado de: https://www.gob.mx/cnbv/acciones-y-programas/bases-de-datos-de-inclusion-financiera

Por otra parte, respecto a otros puntos de acceso, en la Gráfica 3 se observa un aumento en el periodo en corresponsales y cajeros, y particularmente un incremento más considerable en TPVs. En el caso de los cajeros, sí estan contemplados como un indicador de la meta 8.10, siendo el 8.10.1b), y sí ha habido un avance en el periodo en este tipo de infraestructura.

El indicador de corresponsales aumentó en $30 \%$ en el periodo, el de cajeros aumentó en 15\%, mientras que los TPVs aumentaron en 53\%.

Gráfica 3. Cajeros corresponsales y terminales punto de venta por cada 10,000 adultos en México (2015-2020)

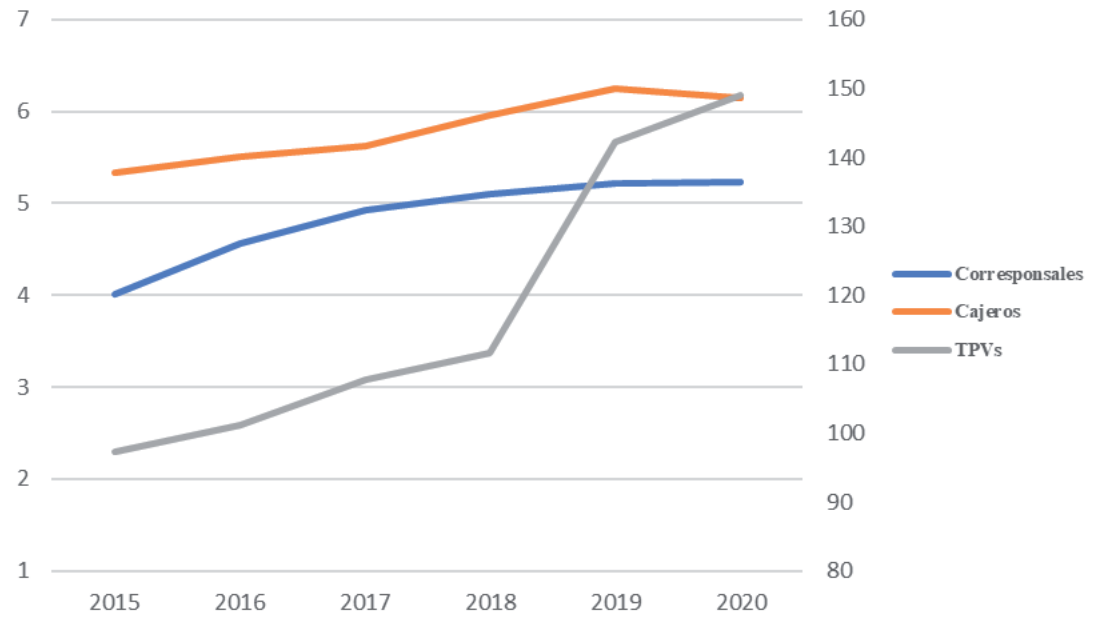


La Gráfica 4 muestra que los contratos de banca móvil han tenido un aumento muy importante en el periodo, en consonancia con la tendencia mundial, y que se ve más pronunciada a partir de 2017 . Aunque estos tipos de contratos no están establecidos como como un indicador de las metas del ODS, promueven la consecución de la meta 8.10. El indicador de contratos que utilizan la banca móvil aumentó un $485 \%$ en el periodo.

Gráfica 4. Contratos que utilizan banca móvil por cada 10,000 adultos en México (2015-2020)

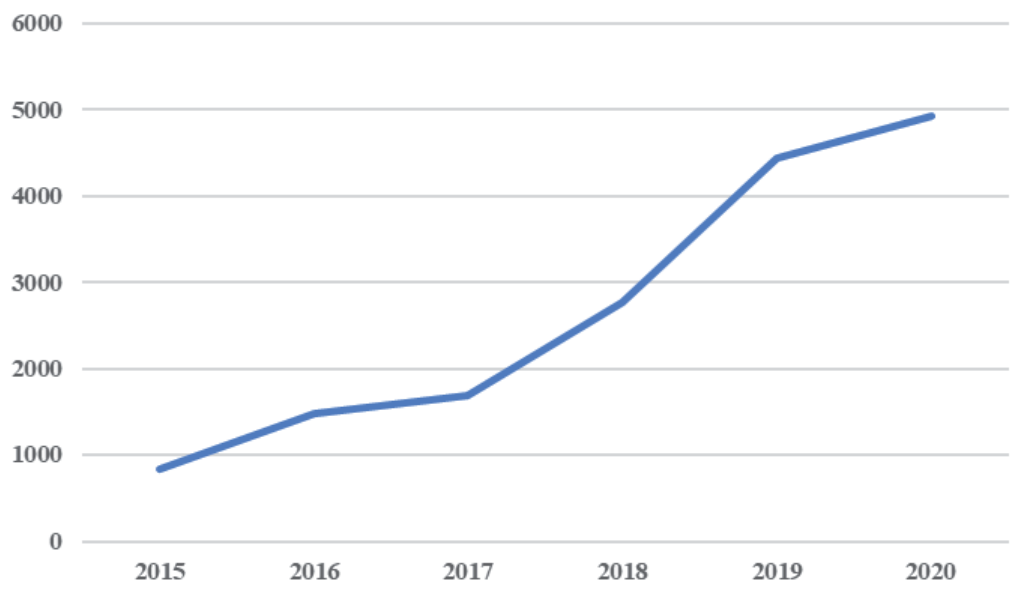

\section{EL SECTOR FINANCIERO Y SU ROL EN TORNO AL CUMPLIMIENTO DE LOS ODS}

\subsection{LOS BANCOS Y EL CUMPLIMIENTO DE LOS ODS: EL INFORME DEL PNUD Y ABM}

En México, el año pasado se realizó una primera evaluación y diagnóstico sobre la contribución de los bancos al cumplimiento de los ODS, a través de una alianza entre el Programa de las Naciones Unidas (PNUD) en México y la Asociación de Bancos de México (ABM) ${ }^{6}$. El PNUD y la ABM desarrollaron y publicaron el Informe de Desarrollo Sostenible 2020 alineado a la Agenda 2030 (en adelante el Informe del PNUD y ABM), en el que se diagnostica específicamente, para cada uno de los ODS y sus metas, las acciones de los bancos que contribuyen al cumplimiento de los ODS. (Villasuso \& PNUD, 2020)

\footnotetext{
${ }^{6}$ La Asociación de Bancos de México representa los intereses generales de la banca en México y actualmente tiene como asociados a 50 instituciones de banca múltiple en el país.

EI PNUD desempeña un papel consultivo fundamental para impulsar la agenda de desarrollo, basándose en las prioridades de los Estados Miembros y el contexto de cada país, y desempeña un papel importante en el fomento de la coordinación dentro del sistema de las Naciones Unidas a nivel nacional, incluyendo la provisión de servicios clave y plataformas de apoyo a los países para apoyar la implementación de los ODS.
} 
De acuerdo con el Informe del PNUD y ABM, la banca en México contribuyó en 2019 en cada uno de los 17 Objetivos de Desarrollo Sostenible, así como a sus metas e indicadores. (Villasuso \& PNUD, 2020) Este informe se realizó con base en una metodología propia del PNUD, que consistió en la creación y desarrollo de un cuestionario que se desarrolló con base en las metas e indicadores de la Agenda 2030, y que fue enviado a las instituciones bancarias a través de ABM. El cuestionario contempló tres aspectos de los bancos: programas de responsabilidad social, productos comerciales y políticas internas de recursos humanos.

El Informe del PNUD y ABM presenta los resultados de las encuestas de los bancos, cuantificando por cada ODS los montos invertidos y la cantidad de personas beneficiadas. En la infografía que se presenta en la Figura 1, tomada del Informe del PNUD y ABM, se ilustra el diagnóstico resumido, para cada uno de los ODS. La cuantificación de la contribución se hace no sólo por objetivos, sino también por metas específicas.

Figura 1. Contribución del sector bancario a cada uno de los ODS de la Agenda 2030

\section{INVERSIÓN}

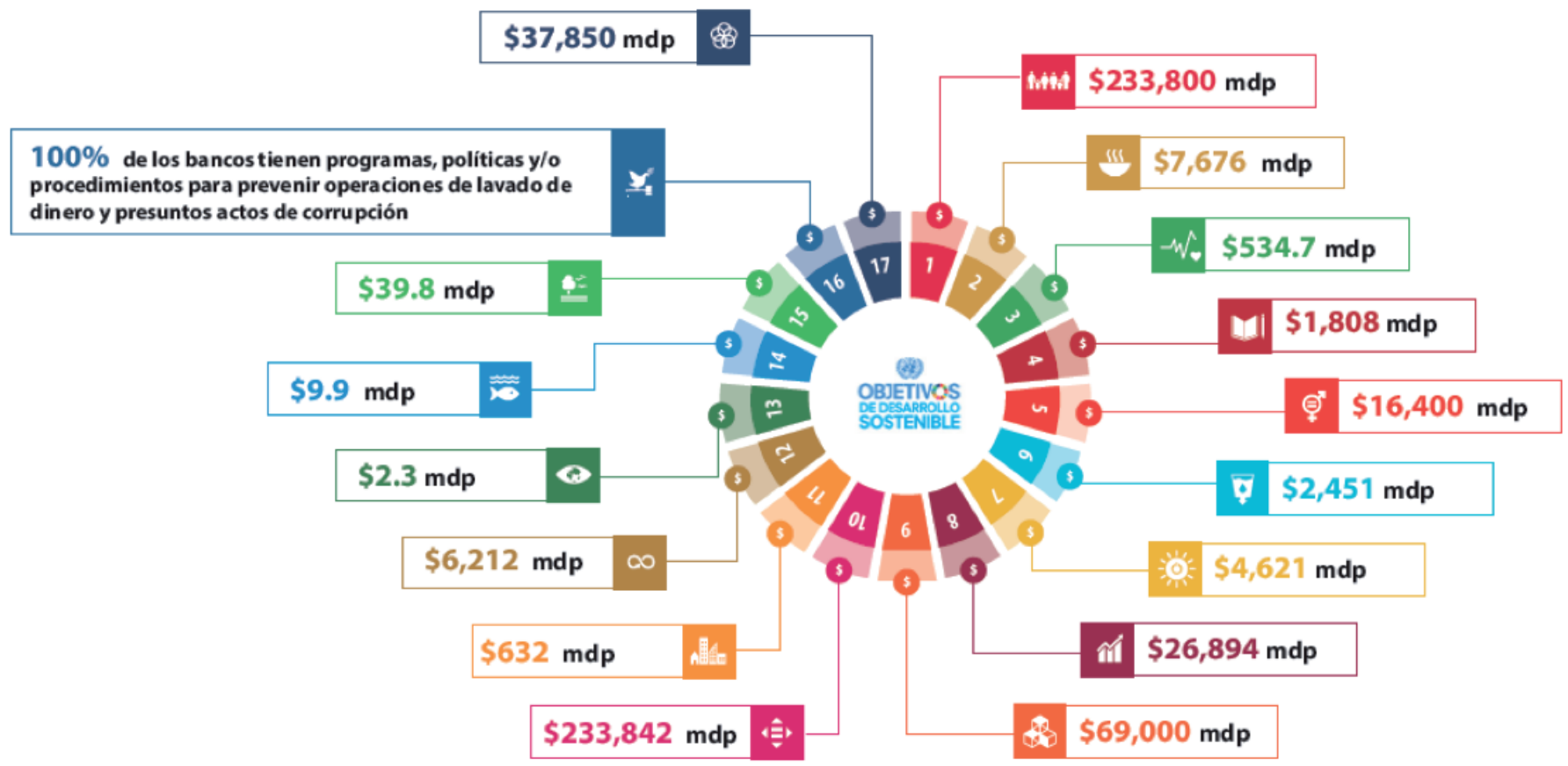

Fuente: Villasuso, M., \& PNUD. (2020). Informe de desarrollo sostenible del sector bancario en México alineado a la agenda 2030. 
De acuerdo a los datos del Informe del PNUD y ABM, la banca en México contribuyó a algunos de los ODS más que a otros, a los que más canalizó sus recursos fueron los siguientes:

ODS 10. Reducción de las desigualdades (233.842 millones de pesos(mdp)) (11.135 millones de euros)7

ODS 1. Fin de la pobreza (233.800 mdp) (11.133 millones de euros)

ODS 9. Industria, innovación e infraestructura (69.000 mdp) (3.2 millones de euros)

ODS 8. Trabajo decente (26.894 mdp) (1.280 millones de euros)

ODS 17. Crecimiento económico y alianzas para Lograr los Objetivos (37.850 mdp) (1.802 millones de euros)

La cuantificación de los montos asignados se hace no sólo por ODS, sino también por metas respectivas. Sin embargo, respecto al ODS 8, que es donde está señalada explícitamente la inclusión financiera, para la meta 8.10, se citan cifras de la infraestructura bancaria total, y cifras de uso, pero no se muestran montos invertidos en expandirla.

\subsection{BONO SOBERANO DE MÉXICO PARA EL FINANCIAMIENTO DE LOS COMPROMISOS DEL ODS}

Una acción reciente de relevancia para el financiamiento sostenible en México es la emisión de un Bono Soberano de los Objetivos de Desarrollo Sostenible (en adelante Bono ODS) de siete años por un valor total de 750 millones de euros, recursos que serán vinculados a programas que contribuyen al logro de los ODS. (PNUD, 2020)

De acuerdo al PNUD, este bono fue pionero al respecto y fue desarrollado con el banco de inversión Natixis, la operación alcanzó una demanda de 4.800 millones de euros, equivalente a 6,4 veces el importe asignado y 267 firmas de inversión globales participaron en la operación.

Seconsideraqueesteesunmecanismoinnovador paraaprovechar el mercadodecapital privadoparafinanciar programas relacionados con los ODS. EI PNUD fue invitado por el Gobierno de México para contribuir a esta iniciativa, emitiendo una opinión en forma de carta de alineación resaltando sus características únicas, incluido el uso de los ODS como punto de entrada y un criterio de elegibilidad que incluye la recopilación de datos espaciales para identificar las comunidades más desfavorecidas. EI PNUD también emitirá una opinión sobre el informe de impacto. (PNUD, 2020)

\footnotetext{
${ }^{7}$ Se hizo la conversión de pesos a euros tomando un tipo de cambio promedio para 2019 de 21 pesos por euro.
} 


\section{CONCLUSIONES Y RETOS HACIA LA INCLUSIÓN FINANCIERA}

En el presente artículo se analizó el vínculo de la inclusión financiera y diferentes aspectos del desarrollo económico, y de esta manera se estableció la importancia de la inclusión para el cumplimiento de los ODS.

Se señala que hay un rezago en la inclusión financiera en México, especialmente en zonas rurales y municipios remotos que carecen de infraestructura. Aunque no solo se trata de un problema de acceso, sino también de uso, como lo evidencia el número de cuentas y otros indicadores de uso para México que se presentaron, que es bajo, comparado con los de la OCDE.

Avanzar en la inclusión financiera en México incidiría para la consecución de los ODS. La inclusión financiera se encuentra explícitamente incluida dentro del ODS 8, en la meta 8.10. Pero además, tal como Klapper et al. (2016) han argumentado y recopilado evidencia empírica al respecto, la inclusión financiera es un medio para conseguir una buena parte de los ODS.

Reconociendo la problemática de la falta de inclusión financiera, en México se han seguido varias estrategias, políticas públicas y cambios en regulaciones, tendientes a promover la inclusión financiera. Destaca la participación de México en la AFI, como miembro de la Declaración Maya, y el establecimiento de un sistema de datos y de medición de la inclusión financiera. Algunas políticas que han promovido la inclusión financiera en México, han sido la introducción y aumento de los corresponsales bancarios, el impulso al sector de ahorro y crédito popular, la introducción de la plataforma CoDi, y el Banco del Bienestar. Todas estas políticas aunque no se refieren a indicadores específicos de los ODS, inciden en la meta 8.10 al promover la inclusión financiera.

Respecto al indicador 8.10.1a, se presentan gráficas en donde se muestra, que hay disminución en el indicador de oficinas en el periodo, mientras que respecto a los cajeros, que también están incluidos como indicador 8.10.1b, hay un avance en el periodo.

Por último se documenta sobre el rol del sector financiero en torno al cumplimiento de los ODS para México. Por un lado, considerando el Informe del PNUD y ABM que es un avance importante al cuantificar los montos con los que contribuyen los bancos a los ODS. Y por otro lado se resalta el que México haya emitido exitosamente un bono alineado a los ODS con el respaldo del PNUD.

El Informe del PNUD y ABM es un esfuerzo importante, sin embargo, hay algunas limitaciones. En el mismo informe se reconoce que los resultados fueron proporcionados por los bancos y no verificados por el PNUD, por lo que se parte de la premisa de que las respuestas brindadas por las instituciones bancarias en el cuestionario son verdaderas. Es necesaria una mayor sistematización para poder medir adecuadamente la contribución del financiamiento de los bancos que incide en los ODS.

Concluyendo, Méxiconecesita hacer mayor esfuerzo paraavanzar en materia de inclusión financieraycumplir plenamente sus compromisos en torno a los ODS. Se requiere que los bancos en México inviertan más en aumentar cobertura de infraestructura bancaria, pues dos de los indicadores de acceso están contemplados en la meta 8.10 de los ODS, y se refieren a la banca comercial. En el caso del indicador 8.10.1a, las oficinas bancarias, hay disminución del indicador en el periodo de $-6 \%$. Para el indicador $8.10 .1 \mathrm{~b}$, referente a los cajeros, hay un aumento en el periodo de $15 \%$.

Considero que se requiere una mayor evaluación del impacto específico de las políticas públicas de inclusión adoptadas. Existen indicadores que miden y dan cuenta de los avances en la inclusión, sin embargo es un tema multidimensional y complejo, y un reto para investigaciones futuras. Se requieren mayores estudios de investigación en México que midan la inclusión financiera y su impacto en variables específicas del desarrollo. 


\section{REFERENCIAS BIBLIOGRÁFICAS}

- Aslan, G., Deléchat, C., Newiak, M. M., \& Yang, M. F. (2017). Inequality in financial inclusion and income inequality: International Monetary Fund.

- Beck, T. (2016). Financial Inclusion - Measuring progress and progress in measuring. This paper was written for the Fourth IMF Statistical Forum "Lifting the Small Boats: Statistics for Inclusive Growth. Cass Business School, City, University of London, CEPR, and CESifo.

- Beck, T., Demirguc-Kunt, A., \& Martinez Peria, M. (2007). Reaching out: Access to and use of banking services across countries. Journal of Financial Economics, 85(1), 234-266. doi:https://doi.org/10.1596/1813-9450-3754

- Bruhn, M., \& Love, I. (2014). The real impact of improved access to finance: Evidence from Mexico. The Journal of Finance, 69(3), 1347-1376. doi:https://doi.org/10.1111/jofi.12091

- CONAIF. (2015). Base de Datos de Inclusión Financiera. Diciembre de 2015. In. México: Consejo Nacional de Inclusión Financiera

- CONAIF. (2016). Base de Datos de Inclusión Financiera. Diciembre de 2016. In. México.

- CONAIF. (2017). Base de Datos de Inclusión Financiera. Diciembre de 2017. In. México: Consejo Nacional de Inclusión Financiera

- CONAIF. (2018). Base de Datos de Inclusión Financiera, Diciembre de 2018. In. México: Consejo Nacional de Inclusión Financiera

- CONAIF. (2019). Base de Datos de Inclusión Financiera, Diciembre de 2019. In. México: Consejo Nacional de Inclusión Financiera

- CONAIF. (2020). Base de Datos de Inclusión Financiera, 2020. In. México: Consejo Nacional de Inclusión Financiera

- CONEVAl. ( 2019). Pobreza y derechos sociales en México. Sistema de indicadores de desarrollo social. Retrieved from Mexico: https://www.coneval.org.mx/Eventos/Documents/2019/Pobreza_derechos_ sociales_Mexico_sistema_indicadores_desarrollo_social.pdf

- Cruz-García, P., Dircio Palacios Macedo, M. d. C., \& Tortosa-Ausina, E. (2021). Financial inclusion and exclusion across Mexican municipalities. Regional Science Policy \& Practice. doi: https://doi.org/10.1111/ rsp3.12388

- Dabla-Norris, M. E., Ji, Y., Townsend, R., \& Unsal, M. F. (2015). Identifying constraints to financial inclusion and their impact on GDP and inequality: A structural framework for policy: International Monetary Fund.

- Del Angel, G. A. (2016). Limits to Cashless Payments and the Persistence of Cash. Hypotheses About Mexico. In E. L. Batiz-Lazo B. (Ed.), The Book of Payments (pp. 117-129). London: Palgrave Macmillan.

- Demirguc-Kunt, A., Klapper, L., \& Singer, D. (2017). Financial inclusion and inclusive growth: A review of recent empirical evidence. Policy Research Working Paper. World Bank Group(8040). doi:https://doi. org/10.1596/1813-9450-8040

- Demirgüç-Kunt Asli, Klapper Leora, Singer Dorothe, Saniya Ansar, \& Jake, H. (2018). The Global Findex Database 2017: Measuring Financial Inclusion and the Fintech Revolution. . Retrieved from: https:// datacatalog.worldbank.org/dataset/global-financial-inclusion-global-findex-database

- Klapper, L., El-Zoghbi, M., \& Hess, J. (2016). Achieving the sustainable development goals. The role of financial inclusion. Available online: http://www. ccgap. org. Accessed, 23(5), 2016.

- Koomson, I., Villano, R. A., \& Hadley, D. (2020). Effect of financial inclusion on poverty and vulnerability to poverty: Evidence using a multidimensional measure of financial inclusion. Social Indicators Research, 1-27. doi: https://doi.org/10.1007/s11205-019-02263-0 
- Levine, R. (2005). Finance and growth: theory and evidence. Handbook of economic growth, 1, 865-934. doi:https://doi.org/10.1016/S1574-0684(05)01012-9

- OECD. (2017). OECD Economic Surveys: Mexico 2017. https://doi.org/10.1787/19990723

- Marco de indicadores mundiales para los Objetivos de Desarrollo Sostenible y metas de la Agenda 2030 para el Desarrollo Sostenible, (2017).

- PNUD. (2020). Bono histórico de 890 millones de dólares para los Objetivos de Desarrollo Sostenible emitido por México [Press release]. Retrieved from https://www.undp.org/content/undp/es/home/newscentre/news/2020/Historic_890_million_SDG_Bond_issued_by_Mexico.html

- Urbina Romero, D. n. (2021). EFICIENCIA DE LOS INTERMEDIARIOS FINANCIEROS NO BANCARIOS DE MÉXICO. Estudios Económicos CNBV, Volumen 4. https://www.gob.mx/cms/uploads/attachment/ file/638272/6._Eficiencia_de_los_IFNB_en_Me_xico.pdf

- Villasuso, M., \& PNUD. (2020). Informe de desarrollo sostenible del sector bancario en México alineado a la agenda 2030. Retrieved from México: https://www.mx.undp.org/content/mexico/es/home/library/ poverty/informe-de-desarrollo-sostenible-del-sector-bancario-en-mexico-a.html 